\title{
Pentadecapeptide BPC 157 antiarrhytmic effect in rats treated with bupivacaine toxic doses
}

\author{
Predrag Sikirić*, Gordana Živanović-Posilović, Diana Balenović, Martina Lovrić Benčić, \\ Sven Seiwerth \\ University of Zagreb School of Medicine, Zagreb, Croatia
}

Introduction: Bupivacaine, still a drug of choice in all of the techniques of regional anaesthesia, acts mainly through blockade of $\mathrm{Na}+$ channels, $\mathrm{K}+$ and $\mathrm{Ca}_{2}+$ channels as well, changing the way the heart conduction system works to cardiac rhythm disturbances in a case of inadvertent i.v. application because we do not have an antidote. Pentadecapeptide BPC 157 antiarrhythmic effects, through its the interaction with NO-system, were already established in the previous studies of digoxin toxicity, hyper- and hypokalemia. The aim of the study is to establish BPC 157 antiarrhythmic effect when cardiac rhythm disturbances are caused by bupivacaine toxic dose and to explore whether this effect is dose-dependent and related with NO-system.

Materials and Methods: The study was conducted on female Wistar albino rats, divided into groups of 6 . We had two protocols with 4 experimental groups each, treated with BPC 157 i.p. (50 $\mu \mathrm{g} / \mathrm{kg}, 10 \mu \mathrm{g} / \mathrm{kg}, 10 \mathrm{ng} / \mathrm{kg}, 10 \mathrm{pg} / \mathrm{kg}$ ) and the control group treated with saline $5 \mathrm{ml} / \mathrm{kg}$ i.p. either 30 minutes before (preventive) or 1 minute after bupivacaine $100 \mathrm{mg} / \mathrm{kg}$ i.p administration (therapeutic). Three standard ECG leads were recorded for 90 minutes. The wave ampli-

\section{Received: 24 ${ }^{\text {th }}$ Apr 2014}

*Address for correspondence: Zavod za farmakologiju Medicinskog fakulteta Sveučilišta u Zagrebu, Šalata 11, HR-10000 Zagreb, Croatia.

Phone: +385-1-4566-833

E-mail: sikiric@mef.hr tudes $P, R, S$ and $T$, the duration of waves and intervals $P$, $P R, Q R S$ and RR, the presence of ventricular ectopies, tachycardia, AV block and asystolia were analyzed.

Results: Bupivacaine caused bradycardia, PQ prolongation, all degrees of AV block, QRS widening, ST-T wave changes, multiform ventricular ectopies (VES), ventricular tachycardia (VT) and asystolia, as well as the decrease of the wave amplitudes. BPC 157 treated animals had in the both protocols and in all of the applied doses less prolongation of $P$ wave in particular $(p<0.001)$, of $Q R S$ complex and $P R, Q T$ and RR intervals as well $(p<0.05)$. The decrease of the wave amplitudes was also counteracted, with lower mortality in the experimental groups (16.7\% vs. $50 \%)$. All of the animals who did not survive had $\mathrm{T}$ wave elevation.

Discussion: BPC 157 has shown cardioprotective effect in the both protocols and in all of the applied doses. Since prolongation of QT interval was lower in the experimental groups, the predisposition to malignant arrhythmias was also lower, which could explain the lower mortality. The T wave elevation present in all of the non survivors is a sign of impeding cardio toxicity and inevitable death, so we can confirm the conclusion of Mauch et al. and propose to use it in clinical practice as an early sign of a threatening catastrophe.

KEYWORDS: cardiac arrhythmias, bupivacaine, pentadecapeptide BPC 157, rats.

CITATION: Cardiol Croat. 2014;9(5-6):217.

\section{Literature}

1. Sikiric P, Seiwerth S, Rucman R, et al. Stable gastric pentadecapeptide BPC 157-NO-system relation. Curr Pharm Des. 2014;20(7):1126-35.

2. Mauch JY, Spielmann N, Hartnack S, Weiss M. Electrocardiographic and haemodynamic alterations caused by three different test solutions of local anaesthetics to detect accidental intravascular injection in children. Br J Anaesth. 2012;108(2):283-9.

3. Barisic I, Balenovic D, Klicek R, et al. Mortal hyperkalemia disturbances in rats are NO-system related. The life saving effect of pentadecapeptide BPC 157. Regul Pept. 2013;181:50-66. 